\title{
A challenging case of SLE associated with intrahepatic cholestasis and atypical Wilson's disease
}

\author{
Jyoti Kankanala*
}

Department of Obstetrics and Gynecology, Citizens Hospital, Hyderabad, Telangana, India

Received: 11 April 2017

Accepted: 08 May 2017

\section{*Correspondence:}

Dr. Jyoti Kankanala,

E-mail: Jyoti.kankanala@gmail.com

Copyright: ( ) the author(s), publisher and licensee Medip Academy. This is an open-access article distributed under the terms of the Creative Commons Attribution Non-Commercial License, which permits unrestricted non-commercial use, distribution, and reproduction in any medium, provided the original work is properly cited.

\begin{abstract}
We herewith present a case of SLE which reflects that appropriate Multidisciplinary approach gives good outcome inspite of risk factors including prematurity. Systemic lupus erythematosus (SLE) an autoimmune disease in itself a high-risk factor along with intrahepatic cholestasis and atypical wilson's disease it's a tough journey for clinicians as well as the family but good timely intervention by appriopriate investigations gave an excellent outcome. Emergency LSCS was performed for pathological CTG at 31 weeks, mother and child followed up for a year and a half then we are reporting this case.
\end{abstract}

Keywords: Autoimmune diseases, Cholestasis, SLE, Wilson's disease

\section{INTRODUCTION}

Systemic lupus erythematosus (SLE) is a chronic inflammatory connective tissue disease commonly diagnosed after the age of 20 , mostly around the age of 30 years. ${ }^{1}$ In $90 \%$ of cases the disease affects women, incidence of SLE during the child bearing age being 1 in 500. Women with SLE are at higher risk for spontaneous abortions, intrauterine fetal death, preeclampsia and eclampsia, preterm delivery and intrauterine growth retardation neonatal lupus, and in extreme cases stillbirth. This paper is a case report of a pregnant woman with SLE not complicated with preeclampsia, IUGR may as on low dose aspirin but other complications existed and managed with team approach.

\section{CASE REPORT}

We report a case of 29 years female with a known case of SLE under the care of a rhematologist and was receiving Tab aziothioprine 50mg and steroid (wysolone) $5 \mathrm{mg}$ and conceived during remission. Remission period gives better outcome. $^{2}$ Azathioprine is one of the only few immunosuppressive agents that has documented safety during pregnancy. ${ }^{3}$ She had a BMI 19, Pulse was 80 beats per minute and a blood pressure of 120/80 mmh. She was started on ecospirin $75 \mathrm{mg}$. Found to be low risk on Combined screening for Down's. MOM for PAPPA was $1.96 \mathrm{mIU} / \mathrm{ml}$. She was under care of rhematologist regularly and was on same medications. At 16 weeks cx length and High Vaginal swab was performed which revealed heavy growth of Streptococcus agalactiac was treated as per sensitivity on culture.

All antenatal investigations including HPLC were within normal limits.Regular urine C3, C4 and microalbumin were performed An important management issue is of recognizing disease flare in pregnant SLE patients. Complement levels rise by $10-50 \%$ during normal pregnancy and may appear to remain in the 'normal' range, despite disease activity. Thus, the trend of complement levels becomes more important than 
absolute values. Mok et al, reported that proteinuria is an important factor that causes fetal loss, our case frequent urine microalbumin was monitored. ${ }^{6}$ S. Anticardiolipin IgM was positive, Anti Ro positive. Patient had anamoly scan and fetal Echo were within normal limits. Received Injection TT and TDAP. At 24 weeks, oral glucose tolerance test was normal and complete blood picture showed mild anaemia was on Iron tablets. Later had complaints of mild vaginal discharge a High vaginal swab revealed candida albicans and treated accordingly. After few weeks came with complaints of severe yellow discolouration of skin and urine, Itching. Detail evaluation by means of biochemistry performed showed abnormal liver function test and total bilirubin being $12.2 \mathrm{mg} / \mathrm{dl}$ by diazo method. AST WAS 39U/L which too was high. Mild anaemia persisted treated with injectable.

Immediately reviewed by Gastroenterologist and rheumatologist and advised to stop aziothioprine and increase the dose of wysolone and was monitoerd frequently and fetal monitoring was performed accordingly and two doses of bethamethasone were adminstered. ANA was negative and 24 hours' urine copper showed 104ug/day. Urine bile salts and bile pigments were positive. All viral screen was negative including hep A and hep E. After a month bilirubin was 1.2 and clotting profile was normal and patient presented with decreased fetal movements and on CTG it was pathological so was taken up for LSCS and MGSO 42 gms given 3 hours prior and she was 31 weeks and 4 days with fetal weight of $1.6 \mathrm{~kg}$ and good apgars and Cord $\mathrm{PH}$ was 7.3 less liquor and thin meconium stained. Inj. oxytocin 40IU in $500 \mathrm{ml} \mathrm{NS}$ was started. The surgery was uneventful with minimal blood loss. After full recovery patient was shifted to the ward and all medications on which patient was maintained antenatally were restarted after 24 hours.Baby needed minimal monitoring baby didn't had any problems of prematurity. It's being 18 months and baby is fine.

Postoperative patient was discharged on day 4 without any antibiotics ro take home and regular gastroenterologist care and wysolne was tappered by this time and rheumatologist was to restart Aziothioprine. Serum ceruloplasmin was not performed by patients on advice as already stressed with so many risk so just wanted to leave it further and we obliged and opthalmic review was done.

\section{DISCUSSION}

An essential component is pre conceptional counseling and disease control prior to pregnancy.

SLE an autoimmune disorder that affects women especially during the reproductive phase has various risk factors like misscarriages, IUD, PET, intrauterine growth restriction, preterm birth. SLE prognosis for both mother and child are best when SLE is quiescent for 6 months and renal and liver functions being stable.
Specific monitoring and treatment protocols are required in high risk situations such as presence of specific antibodies (aPL and anti-Ro). Low dose aspirin alone is recommended for asymptomatic women with only persistently positive aPL and no prior event. ${ }^{4,5}$

Neonatal lupus erythematosus (NLE) is a syndrome characterized by skin, cardiac, and systemic abnormalities seen in newborn infants whose mothers have autoantibodies against Ro/SSA and/or La/SSB. ${ }^{7}$ The condition was first described almost 60 years ago by McCuistion and Schoch, who noted the presence of characteristic skin lesions on a baby born to a mother who suffered from lupus. ${ }^{8}$ By the late 1970s, NLE was found to be caused by maternal transplacental passage of the anti-SSA/Ro antibodies from mother to fetus. ${ }^{9}$ The term NLE was initially selected because of the similarity of skin lesions with those that can occur in subacute cutaneous lupus. However, the disparities between the NLE and those living with systemic lupus, especially for cardiac involvement, highlight the inappropriateness of the term, and only minorities of mothers have defined systemic lupus erythematosus. Furthermore, many of the mothers have or go on to develop primary Sjogren's syndrome or undifferentiated autoimmune disease, and many of them are totally asymptomatic. This article will provide an overview of NLE including clinical presentations, pathogenesis, and treatment. Much of the current knowledge about this syndrome based on the study of the American Neonatal Lupus Registry and a recent large French study.

Neonatal lupus is rare is associated with Maternal anti-Ro antibodies. Not all pregnancies in the setting of anti Ro/LA antibodies are associated with Congenital Heart Block so prophylactic treatment is not recommended only 2-D ECHO can be performed post 4 weeks.

Intrahepatic Cholestasis common among south asians, the worrisome part is sudden IUD due to taurocholate crossing in to the fetal compartment and causing fetal arrythmias and decreased contractility or chorionic vein constrictiondue to Bile Acid cholate.

Wilsons disease with increase in 24 hours' urine copper was worrisome and patient wanted to avoid further investigations as very few cases in literature about standard care.

\section{CONCLUSION}

Detailed Clinical evaluation of pregnant women with SLE is a must with preanaesthetic evaluation as well as early as possible and she will be labelled as high risk. Timely intervention can prevent major morbidity and mortality. Pregnancy can be successful in all aspects if well planned so as great social, financial support can be sorted. As she would need frequent visits and treatment as per the complications which yet times can be 
unexpected so a compassionate continuity of care pathway should be established.

Funding: No funding sources

Conflict of interest: None declared

Ethical approval: Not required

\section{REFERENCES}

1. Mills JA. Systemic Lupus Erythematosus. N Engl J Med. 1994;330:1871.

2. Lateef A, Petri M. Management of pregnancy in systemic lupus erythematosus. Nat Rev Rheumatol. 2012;8(12):710-8

3. Ostensen M, Khamashta M, Lockshin M, Parke A, Brucato A, Carp H, et al. Anti-inflammatory and immunosuppressivedrugs and reproduction. Arthritis Res Ther. 2006;8(3):209

4. Del Ross T, Ruffatti A, Visentin MS, Tonello M, Calligaro A, Favaro M, et al. Treatment of 139 pregnancies in antiphospholipid positive women not fulfilling criteria for antiphospholipid syndrome: a retrospective study. J Rheumatol. 2013;40(4):425-9.

5. Petri M, Qazi U. Management of antiphospholipid syndrome in pregnancy. Rheum Dis Clin North Am. 2006;32(3):591-607.

6. Hayslett JP, Lynn RI. Effect of pregnancy in patients with lupus nephropathy. Kidney Int 1980;18:207-20.

7. Buyon JP, Rupel A, Clancy RM. Neonatal lupus syndromes. Lupus 2004;13:705-12.

8. Lee LA. The clinical spectrum of neonatal lupus. Arch Dermatol Res. 2009;301:107-10.

9. Silverman E, Jaeggi E. Non-cardiac manifestations of neonatal lupus erythematosus. Scand J Immunol. 2010;72:223-5.

Cite this article as: Kankanala J. A Challenging case of SLE associated with Intrahepatic Cholestasis and atypical wilson's disease. Int J Reprod Contracept Obstet Gynecol 2017;6:2673-5. 